\title{
Features of elastic scattering at small $t$ at the LHC
}

\author{
O.V. Selyugin* and J.-R. Cudell ${ }^{\dagger}$ \\ * Bogoliubov Laboratory of Theoretical Physics, JINR, 141980, Dubna, Russia \\ ${ }_{\dagger}^{\dagger}$ IFPA, Dept. AGO, Bât. B5a, Université de Liège, B4000 Liège, Belgium
}

\begin{abstract}
The problems linked with the extraction of the basic parameters of the hadron elastic scattering amplitude at the LHC are explored. It is shown that one should take into account the saturation regime which will lead to new effects at the LHC.
\end{abstract}

Keywords: elastic scattering, LHC energy

PACS: $11.80 . \mathrm{Cr}, 12.40 . \mathrm{Nn}, 13.85 . \mathrm{Dz}$

In the nearest future, new experiments will measure the elastic scattering amplitude at the LHC. We hope that they will improve the theoretical understanding of this basic object of hadronic physics. Now models predict a wide range of possibilities, for example for the growth of $\sigma_{t o t}(s)$. However, we had such a situation a long time ago.

"With the construction of large accelerators, it is hoped that the mysteries of high-energy scattering will unfold in the near future."

Hung Cheng, Tsai Tsun Wu, Phys. Rev. Lett. (1970).

Long after, we met the problems linked with different results for the size of $\rho$, the ratio of the real part to the imaginary part of the scattering amplitude [UA4 measured $\rho=0.24$ and UA4/2 $\rho=0.139]$ and then, at the Tevatron, $\sigma_{t o t}(s)$ is ill-determined $\left[\sigma_{t o t}=(71.42 \pm 2.41) \mathrm{mb}\right.$, the results of E811 experiment or $\sigma_{t o t}=(80.03 \pm 2.24) \mathrm{mb}$ according to CDF.1]

The theoretical predictions for $\sigma_{t o t}$ at the LHC cover a wide range, from $80 \mathrm{mb}$ up to $230 \mathrm{mb}$. For elastic scattering, several models predict very different behaviours, such as, for example, oscillations, or a non-exponential dependence of $d \sigma / d t$ on $t$ [1]. These uncertainties are usually connected with the interaction at large distances.

Furthermore, it was shown [2, 3] that, at small impact parameter $b$, the overlapping function grows and may reach a saturation regime at the LHC, which leads to essential changes in the $t$-dependence of $\rho$ and of the slope $B$. Hence, the measurements of forward elastic scattering will give us information on hadron interactions at small $b$ and large distances.

\footnotetext{
${ }^{1}$ Note that it is likely that this difference reflects the true errors of the procedure extracting $\sigma_{t o t}$ from the experimental data through the luminosity-independent method.
} 

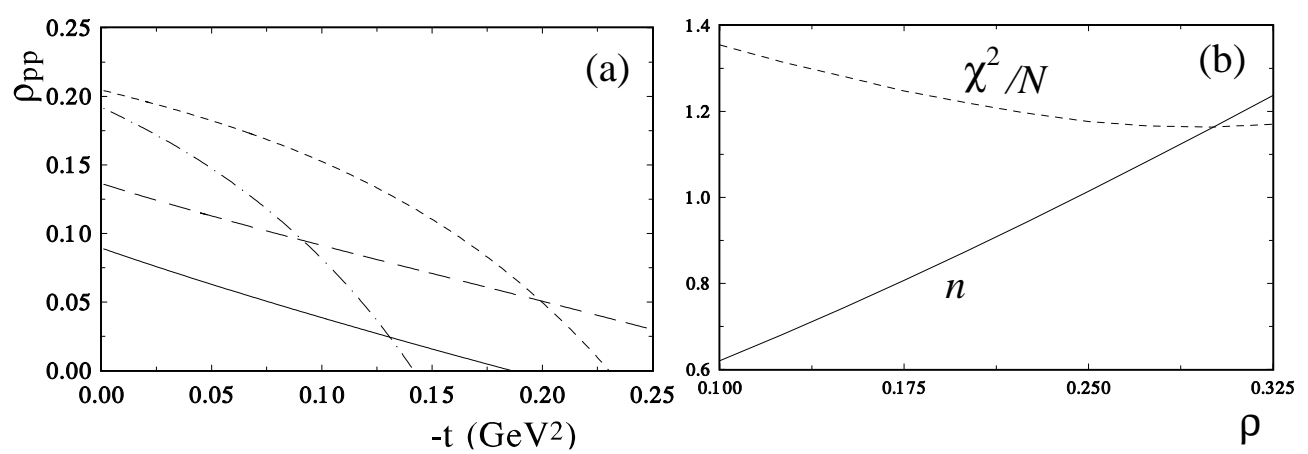

FIGURE 1. (a) The ratio $\rho(s, t)$ in the Dubna Dynamical Model [4] at $\sqrt{s}=100 \mathrm{GeV}$ (plain curve), $500 \mathrm{GeV}$ (long dashes), $5 \mathrm{TeV}$ (short dashes) and $\sqrt{s}=14 \mathrm{TeV}$ (dash-dotted curve); ( b) The dependence on $\rho$ of $\chi^{2} / N$ and of the normalisation coefficient $n$ obtained in the fitting procedure of the simulated data at $\sqrt{s}=14 \mathrm{TeV}$.

\section{DETERMINATION OF $\sigma_{\text {tot }}(\mathbf{s})$ AND $\rho(\mathbf{s}, \mathbf{t})$}

The experimental analyses of the differential elastic cross section usually rely on the following assumption:

$$
\operatorname{ImF}(s, t) \sim \operatorname{ReF}(s, t) \sim e^{B t / 2}
$$

Let us note that in the fitting procedure of $d \sigma / d t$, one must vary at least four parameters, $\sigma_{t o t}(s), B(s, t), \rho(s, t)$ and $n$, the normalisation coefficient reflecting the systematic errors (the fitted luminosity is $n$ times the true one). Eq. (1) works reasonably well at low energies. At high energies, the elastic amplitude becomes a sum of many different diagrams which unitarize it and this simple behaviour changes.

In some models [3, 4], which describe the differential cross section in a wide region of $s$ and $t$, the amplitude reaches the Black Disk Limit (BDL). This leads to a significant change of the $t$ dependence of $\rho$ and $B$, as seen in Fig. 1.a and [2]. In this case, the standard fitting procedure by an exponential will give bad results. To see this, we can simulate data at $\sqrt{s}=14 \mathrm{TeV}$ from the model of ref. [4], and then analyse them using an exponential form for the scattering amplitude. In Fig. 1.b, we show the result: large differences in $\rho$ and $n$ cannot be detected by a change in $\chi^{2}$.

As a consequence, one can obtain very wide variations of $\sigma_{\text {tot }}$ depending on the input $\rho$, as shown in Fig. 2.a. But the value of $\rho$ at $\sqrt{s}=14 \mathrm{TeV}$ strongly depends on the model, so it must be included in the fitting procedure.

Note also that the slope of the differential cross sections does not necessarily grow with $s$ [5]. Some models predict a non-vanishing spin-flip amplitude at high energies. Such a contribution to the differential cross section will lead to a decrease of the slope as $t \rightarrow 0$. Indeed, let us assume that the slope of the spin-flip amplitude, $F_{s f} / \sqrt{|t|}(t=$ $0)=\tau F_{s n f}(t=0)$, is $\Delta B / 2$ larger than the slope $B_{0}$ of the spin-non-flip amplitude. In this case, we have for the total slope [6]

$$
B(t)=B_{0}-\frac{2 \tau^{2}(1+t \Delta B)}{m^{2}+2 \tau^{2}|t| \exp (\Delta B t)} e^{\Delta B t},
$$



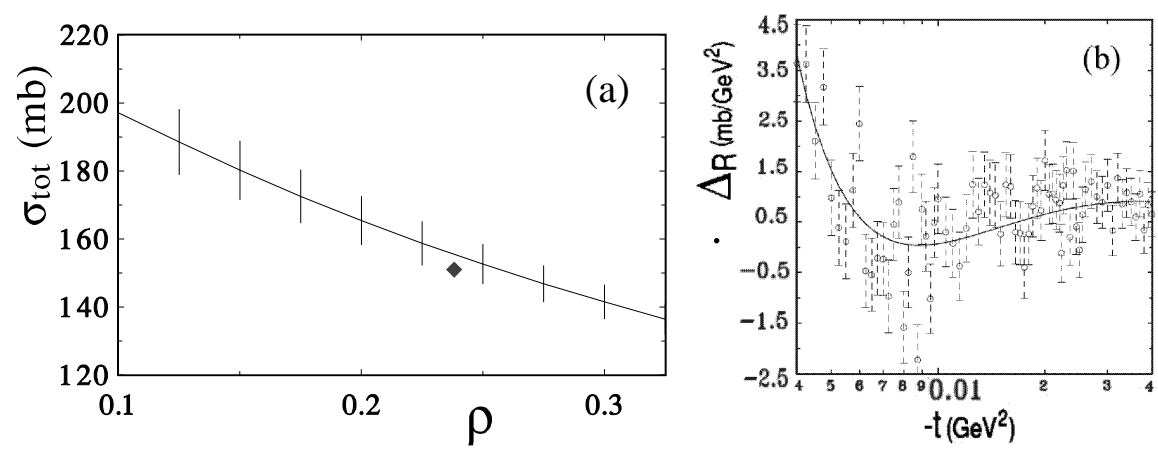

FIGURE 2. (a) $\sigma_{t o t}$ obtained from the standard fitting procedure using an exponential form of the amplitude and a fixed $\rho$ at $\sqrt{s}=14 \mathrm{TeV}$ with free normalization $(n)$; the diamond gives the input $\sigma_{\text {tot }}$ and $\rho$; (b) $\Delta_{R}$ obtained from the simulated data at $\sqrt{s}=14 \mathrm{TeV}$ analysed with an exponential form of the scattering amplitude(points with errors); the curve gives the model prediction with $\rho=0.15$.

and for the cross section:

$$
\frac{d \sigma}{d t}=\frac{1}{16 \pi}\left(1+\rho^{2}\right) \sigma_{\text {tot }}^{2}\left(1-2 \tau^{2} \frac{t}{m^{2}} e^{\Delta B_{0} t}\right) e^{B_{0} t} .
$$

\section{FEATURES OF THE t-DEPENDENCE OF THE REAL PART}

In order to measure reliably $\sigma_{t o t}$, we need to find additional ways to determine $\rho(s, t)$. One possibility was given in [7], which uses a particular property of the proton-proton scattering amplitude at high energies and small $t$ : at a specific value $t=t_{R}$, the real part of the Coulombic amplitude cancels the real part of the hadronic amplitude $\operatorname{ReF}_{C}\left(t_{R}\right)+$ $\operatorname{ReF}_{h}\left(t_{R}\right)=0$. So, the value $\Delta_{R}=\left(R e F_{C}+R e F_{h}\right)^{2}$ will have a minimum at this special point, and the value of $t_{R}$ may be possible to extract from the experimental data. Note that this is a generic property that does not rely on a given model. One has in general

$$
\begin{aligned}
\Delta_{R}(s, t) & =\left[\operatorname{ReF}_{h}(s, t)+\operatorname{Re}_{C}(s, t)\right]^{2} \\
& =\left[(1 / \pi) d \sigma^{\exp } / d t-\left(\alpha \varphi F_{C}(t)+\operatorname{ImF}_{h}(s, t)\right)^{2}\right] .
\end{aligned}
$$

We show in Fig. 2.b what such an analysis might give, for the simulated data at $\sqrt{s}=$ $14 \mathrm{TeV}$, fitted by a simple exponential form with $\rho=0.15$.

There can be some additional effects which change the form of $d \sigma / d t$. For example, there can be some small oscillations in the scattering amplitude which are predicted by several models (see [1] for a review ). One such possible oscillation with period proportional to $q=\sqrt{|t|}$ was analyzed in [8]. It was shown that such oscillations may exist in the experimental data obtained at $\sqrt{s}=541 \mathrm{GeV}$, as shown in Fig. 3.a. However in the standard fitting procedure, if we do not now the form and $t$-dependence of such oscillations, they are hidden in the statistical noise. In [8], a new method was proposed, based on statistically independent choices. The experimental interval in $\sqrt{ }|t|$ is divided 

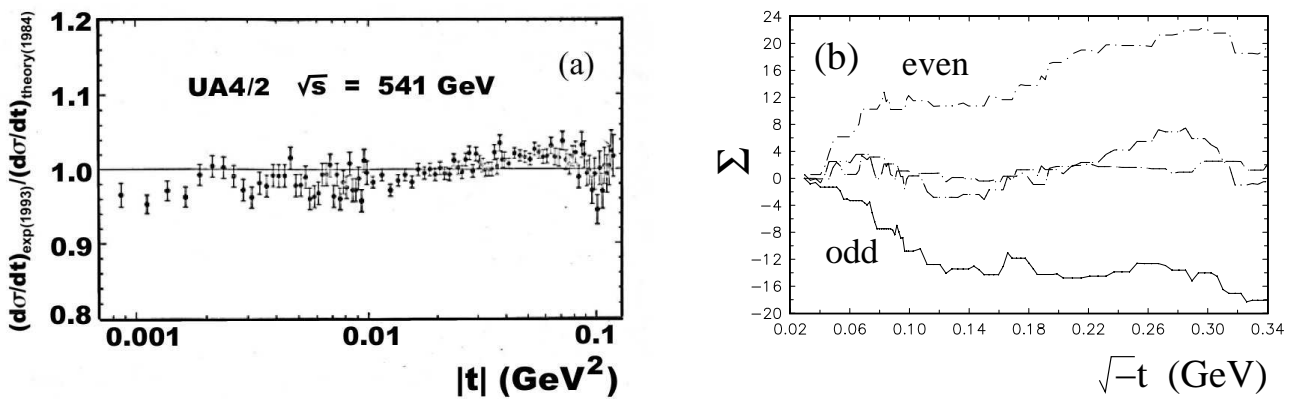

FIGURE 3. (a) Ratio of the experimental data to the exponential description at $\sqrt{s}=541 \mathrm{GeV}$; (b) The statistical sums (upper and lower curves) of the even and odd intervals coincides with possible oscillations; the middle curves are the same sums but the beginning of the intervals was shifted by half an interval.

into small equal intervals $\delta_{i}$. One then calculates sums of the differences between the experimental data and the theoretical curve normalised by the experimental error, for $i$ odd or even:

$$
\Sigma^{\text {odd }(\text { even })}=\sum_{i \text { odd }(i \text { even })} \frac{d \sigma / d t_{i}^{\text {data }}-d \sigma / d t_{i}^{\text {theory }}}{\sigma_{i}}
$$

The corresponding curves are shown in Fig. 3.b. The statistical analysis has shown the existence of oscillations with a period $\sim \sqrt{|t|}$ at a significance level of $3 \sigma$.

We can conclude that some additional research is needed. It is likely that the BDL regime will be reached at LHC energies. Its effects will have to be taken into account and one will have to fit simultaneously all 4 parameters $n, \sigma_{t o t}, B(s, t)$ and $\rho(s, t)$. To investigate the non-linear behaviour of the parameters of the scattering amplitude, one will probably need to develop new methods.

Acknowledgments: O.V. Selyugin is grateful to the Organizing Committee of the conference for the financial support and the warm hospitality, and also wishes to acknowledge support from FNRS and from the Université de Liège where part of this work was done.

\section{REFERENCES}

1. R. Fiore et al., arXiv:0810.2902 [hep-ph].

2. J. R. Cudell and O. V. Selyugin, Czech. J. Phys. 54 (2004) A441 [arXiv:hep-ph/0309194].

3. J. R. Cudell and O. V. Selyugin, Phys. Lett. B 662 (2008) 417 [arXiv:hep-ph/0612046].

4. S. V. Goloskokov, S. P. Kuleshov and O. V. Selyugin, Z. Phys. C 50 (1991) 455; Sov. J. Part. Nucl. 18 (1987) 14.

5. O. V. Selyugin, Phys. Lett. B 333 (1994) 245 [arXiv:hep-ph/9312305].

6. O. V. Selyugin, Mod. Phys. Lett. A 14 (1999) 223.

7. P. Gauron, B. Nicolescu and O. V. Selyugin, Phys. Lett. B 629 (2005) 83 [arXiv:hep-ph/0406177].

8. O. V. Selyugin, in the proceedings of the International Workshop on High-energy Physics: Diffraction 95, Novy Svet, Ukraine, 6-11 Sep 1995 p. 65.; Ukr. J. of Phys. 3 (1996) 296; P. Gauron, B. Nicolescu and O. V. Selyugin, Phys. Lett. B 397 (1997) 305. 